\section{An interview with Alfredo Falcone and Lisa Salvatore: RECOURSE and trifluridine/tipiracil in metastatic colorectal cancer}

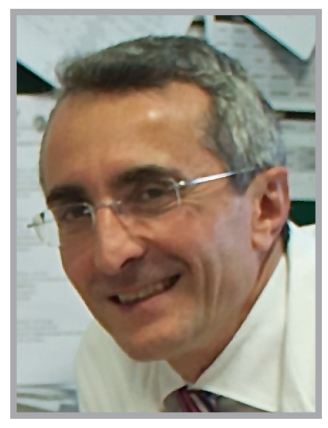

Professor Alfredo Falcone ${ }^{*, 1}$ and Dr Lisa Salvatore ${ }^{1}$ speak to Roshaine Gunawardana, Managing Commissioning Editor: Professor Alfredo Falcone is the Director of the Department of Oncology and the Specialization School at the University Hospital of Pisa, Italy. He trained in Pisa and Genoa, Italy, and has held major positions in Italian oncology since 2000. He currently has more than 300 publications, including papers in peer-reviewed international and national journals, book chapters, and more than 600 abstracts of presentations to international and national conferences. The majority of his papers

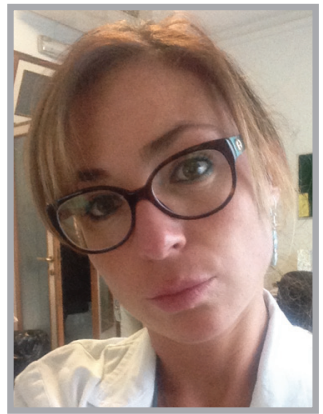
regard clinical and translational research, with a particular focus on metastatic colorectal cancer. Dr Lisa Salvatore is a medical oncologist in the Department of Translational Research and New Technologies in Medicine and Surgery at the University of Pisa. She has been an author on about 40 publications in major peer-reviewed publications and has made numerous presentations in national and international conferences. Her main interest is focused on clinical and translational research in metastatic colorectal cancer.

First draft submitted: 25 May 2016; Accepted for publication: 25 May 2016; Published online: 7 June 2016

In this interview, Alfredo Falcone and Lisa Salvatore talk about their careers and their vision of research in colorectal cancer. They describe their roles in the clinical development of trifluridine/tipiracil for the management of metastatic colorectal cancer and their participation in the RECOURSE trial.

Q Can you tell us about your careers? How did you become involved in research in colorectal cancer?

- Alfredo Falcone

My career began at the University of Pisa, where I received my degree in Medicine in 1984. In subsequent years, I worked at the Istituto Scientifico Tumori in Genoa, where I received my specialization in Oncology, and then from 1987 to 1989 at the Division of Oncology/Hematology at the Roger Williams Cancer Center, Brown University (RI, USA). During these years, I became involved in translational and clinical research in oncology, focusing primarily on cancers of the colon-rectum and gastrointestinal. Today I am Professor of Medical Oncology at the University of Pisa and Head of the Medical

'University of Pisa, Azienda Ospedaliero Universitaria Pisana, Istituto Toscano Tumori (ITT), Via Roma, 67, 56126 Pisa, Italy *Author for correspondence: Tel.: +39050 992466; +39050 992111; alfredo.falcone@med.unipi.it

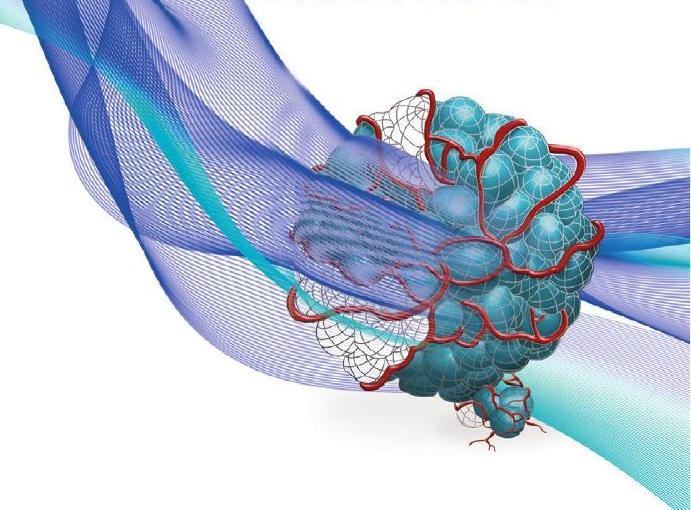

(n)

(1)


Oncology Unit at the University Hospital of Pisa (Italy). I am also chairman of the National Cooperative North-West Oncology Group (GONO).

\section{- Lisa Salvatore}

My career hasn't been so long! I started to work with the Oncology Unit directed by Professor Falcone in Pisa in January 2005 during my fifth year at University. I obtained my degree in Medicine in July 2007 and, in March 2008, I started my Fellowship in Oncology at the University of Pisa with Professor Falcone, which lasted until April 2012. In November 2014, I started a Post-Doctoral Fellowship in Clinical Pathophysiology at the University of Pisa. I'm still working at the University of Pisa with Professor Falcone, and I'm involved not only in clinical practice, notably in colon cancer, but also in clinical and translational research for colorectal cancer.

\section{Q What would you say were your greatest achievements to date? \\ - Lisa Salvatore}

Well, I think it's to have witnessed the birth of FOLFOXIRI (folinic acid, fluorouracil [FU] oxaliplatin, and irinotecan) as first-line treatment in metastatic colorectal cancer. Indeed, Professor Falcone could be regarded as the father of FOLFOXIRI. The main success was the Phase III TRIBE study (Triplet plus Bevacizumab) that demonstrated the superiority of FOLFOXIRI plus bevacizumab versus FOLFIRI (folinic acid, fluorouracil, and irinotecan) plus bevacizumab in the first-line treatment of metastatic colorectal cancer. The results in terms of outcomes were sufficiently promising for a publication in the New England Journal of Medicine and in the Lancet Oncology [1,2]. I think this is one of the greatest achievements not only for the group directed by Professor Falcone, but also for Italian no-profit research.

The other main achievement of our group has been the participation in the RECOURSE (Randomized, double-blind, Phase 3 study of TAS-102 plus best supportive care [BSC] versus placebo plus BSC in patients with metastatic colorectal cancer refractory to standard chemotherapies) trial, which tested trifluridine/tipiracil (TAS-102) versus placebo in metastatic colorectal cancer. These results were also published in the New England Journal of Medicine [3]. Our group was one of the first enrolling centers in the world. The results led to the US FDA and European approval of the drug. I think that we can be very proud of this!

\section{Q How has the management of metastatic} colorectal cancer changed over the last decade?

The management of metastatic colorectal cancer has improved enormously over the last decade, and it is still improving and it will improve in the future. This is due to the number of advances, including the introduction of new drugs, such as biological drugs; the personalization of treatment, e.g., anti-EGFR monoclonal antibodies; the introduction of new combinations of chemotherapeutic agents; and new treatments such as trifluridine/tipiracil. There has also been an improvement in techniques for radical surgery of metastatic sites, as well as an improvement in the multidisciplinary discussion between oncologists, nurses, radiotherapists, surgeons, and other players in the patient journey. This last point is especially important. Indeed, I think that the management of metastatic colorectal cancer has to be considered in terms of a multidisciplinary team. We have to personalize the treatment and strategy for each patient.

Thanks to this progress, we have also seen a substantial prolongation of survival. We have moved from 1 year of survival up to more than 30 months, and even more than 40 months in overall survival in specific selected patients. And so yes, I think that we have prolonged overall survival with a good quality of life. I think it is very important to not only prolong survival, but also to improve or maintain a good quality of life.

Q What are the treatment options in the later stages of metastatic colorectal cancer?

There are now more treatment options for the later stages of metastatic colorectal cancer than in the last few years. Firstly, there is the rechallenge with standard active chemotherapeutic drugs to choose on the basis of previously observed benefits and toxicity, even if there are no prospective clinical studies on rechallenge and evidence is weak. More essential, we now have two new drugs - regorafenib and trifluridine/tipiracil - which are proving active in the specific setting of patients that are previously treated and refractory to all standard treatments. 
Q You have been an active participant in the clinical development of the new agent trifluridine/tipiracil. How is this different from other antimetabolites, such as 5-FU \& capecitabine?

There are both similarities and differences between trifluridine/tipiracil and 5-FU and capecitabine, which breaks down into 5-FU. First, both drugs are incorporated into tumor cells as thymidine analogues and their metabolites can be incorporated into DNA with double-chain damage. The induction of DNA dysfunction is the major route for activity of trifluridine/tipiracil, while 5-FU leads to inhibition of DNA synthesis. Preclinical data have shown that trifluridine (FTD), i.e., the active component of trifluridine/tipiracil, has a markedly greater degree of incorporation into DNA compared with 5-FU, and also that the incorporation of FTD in tumor tissues is significantly higher than its incorporation into normal tissues [4,5]. Furthermore, FTD and 5-FU seem to arrest the cell cycles in different steps. Interestingly, the polymorphisms of dihydropyrimidine dehydrogenase (DPD) are not relevant for FTD metabolism.

Second, the two drugs are also similar as they both inhibit thymidylate synthase (TS). Here, however, we have an important difference: while the active metabolite of 5-FU inhibits TS irreversibly, the active metabolite of trifluridine/tipiracil blocks TS reversibly. Indeed, the mechanism of action of FTD appears to goes beyond TS inhibition, which is a relatively minor route.

Finally, trifluridine/tipiracil is a combination drug, and so we need to consider the effector, tipiracil (TPI). TPI is essential for two main reasons: it prevents the rapid degradation of FTD and there are preclinical data suggesting that it may have a direct role as an antiangiogenic factor [4]. And so we may have a double action for TPI.

Altogether, trifluridine/tipiracil has a distinct mode of action from 5-FU, and appears as a new treatment option in pretreated patients, including those with acquired resistance or intolerance to the other antimetabolites.

\section{Q Can you tell us more about the RECOURSE trial?}

RECOURSE was a double-blind, placebo-controlled Phase III trial. RECOURSE was a truly international trial, with centers in Japan, USA, Europe and Australia. Italy was a major player, with nine actively participating centers. We enrolled metastatic colorectal cancer patients, who had previously been treated with, or were not considered candidates for, all available active drugs for their disease, such as fluoropyrimidines, oxaliplatin, irinotecan and bevacizumab, and cetuximab or panitumumab for $K R A S$ wild-type patients.

A total of 800 patients were enrolled in RECOURSE with a randomization at a ratio of 1:2 to receive a placebo or trifluridine/tipiracil. The primary end point was overall survival. The study was positive - the primary end point was met. Treatment with trifluridine/tipiracil reduced the risk of death by more than $30 \%$ versus placebo (hazard ratio: 0.68 ; 95\% CI: $0.58-0.81$; $\mathrm{p}<0.001)$ with an absolute gain in median overall survival of about 2 months versus placebo. The rates of overall survival at 1 year were $27 \%$ and $18 \%$, respectively. We need to consider not only the significant improvement in overall survival, but also improvements in secondary end points such as progression-free survival. In RECOURSE, we saw a significant improvement in progression-free survival with a reduction of risk of progression of more than 50\% (hazard ratio: $0.48 ; 95 \% \mathrm{CI}: 0.41-0.57$; $\mathrm{p}<0.001$ ). There was also a significant improvement in rate of disease control - that is, complete or partial response or stable disease, with a rate $44 \%$ with trifluridine/tipiracil versus $16 \%$ with placebo ( $p<0.001)$. The results of the RECOURSE trial were published in 2015 [3], and these results led to the regulatory approval of the drug in the USA and, very recently, in Europe.

\section{Q Why did the overall survival result for trifluridine/tipiracil cause so much excitement?}

There is much reason to be excited when we consider the absolute gain in survival! It could be argued that we have obtained only 2 months in overall survival, but, considering the setting of the patients, I think that this is a great result! We should recall that these are patients who had limited alternatives for treatment beyond BSC, and that they were refractory to all standard treatments. Discovering a new drug that can significantly improve overall survival in such patients is simply a great result. We should also consider that trifluridine/tipiracil decreased the risk of death by more than $30 \%$ and the risk of progression by more than $50 \%$, which are very good results. 
Next, we need to consider the safety profile: this is a well-tolerated drug. The most frequent toxicities with trifluridine/tipiracil are hematological toxicities, in particular neutropenia and anemia. In RECOURSE, 38\% of patients experienced grade 3 or 4 neutropenia, but only $4 \%$ had febrile neutropenia. This means that the neutropenia could be an adverse event, but could also be just a laboratory value because there is no increase in complicated neutropenia. Anemia was also a frequent adverse event with trifluridine/tipiracil, with about $18 \%$ of grade 3 or 4 anemia versus $3 \%$ in the placebo group. The incidence of any grade 3 or 4 adverse events or serious adverse events was quite similar between trifluridine/tipiracil and placebo, and adverse events resulted in study withdrawal in $4 \%$ of trifluridine/tipiracil patients versus $2 \%$ of placebo patients. Therefore, if we consider the overall safety profile, we can conclude that it is a well-tolerated drug.

Q Concretely, what do the RECOURSE results mean for patients?

I think that they mean that patients have a new chance for treatment. Even though this is a palliative setting, we often see patients presenting in very good general condition. A new treatment option can provide further hope for prolonging the overall survival of these patients. The clinical impact is relevant in terms of progression-free survival, disease control rate, and also prolongation of overall survival. The good safety profile is also important. The RECOURSE trial did not include formal collection of data on quality of life. However, we can surmise that the manageable safety profile would have a positive impact on quality of life. Such considerations may explain, in part, the longer delay to the worsening of ECOG (Eastern Cooperative Oncology Group) performance status $\geq 2$ in patients treated with trifluridine/tipiracil (5.7 months vs 4.0 months with placebo; $\mathrm{p}<0.001)$.

Q Is a particular patient profile that would benefit most from trifluridine/tipiracil?

There are no formal data on particular patient profiles, though we should consider that we now have two different treatments for the later stages of metastatic colorectal cancer - regorafenib and trifluridine/tipiracil - with a completely different mechanism of action and a completely different safety profile, but with a similar efficacy. There is also a lack of data to choose the best treatment for each patient or the best sequence, and so how we can choose the best treatment is quite difficult. We need to consider the toxicity profile of the agents, previous treatments, and previous toxicities. We also need to consider the main selection criteria of the various clinical trials, because the main difference in my opinion is the definition of refractory patients. We are waiting for biomarkers and translational studies, hoping that future studies will provide something that can help us make the right choices.

\section{Q Could you comment on the time before} deterioration of these patients?

One of the secondary end points of the RECOURSE trial was the delay to the worsening of ECOG performance status from the baseline of zero or one, to a score of two or higher. The results of the RECOURSE trial demonstrated that median time to an ECOG performance status of two or higher was 5.7 months in the experimental arm and 4.0 months in the placebo group with a hazard ratio of $0.66(\mathrm{p}<0.001)$. I think that this is an important result because performance status indirectly reflects the improvement in the efficacy of trifluridine/tipiracil on progressionfree survival, disease control rate and also overall survival, as well as its good safety profile.

\section{Q Can you tell us more about your} experience of the use of this product in clinical practice?

We have treated a lot of patients with trifluridine/ tipiracil, not only in RECOURSE trial but also now as part of the compassionate use program, in which we have treated more than 100 patients. It is very reassuring that the safety we observed in the compassionate use program is very similar to what we observed in the RECOURSE trial. We also see a very similar clinical activity of trifluridine/tipiracil in terms of disease control, delay to deterioration of performance status, improvement in the delay to progression of disease, and improvement in overall survival. The safety aspects are the same. There are no specific precautions for use: we just need to pay attention in patients with low hemoglobin levels because the problem of anemia can be important, and sometimes blood transfusion is necessary.

\section{Q What do you see as the next steps in the development for trifluridine/tipiracil?}

I think the next step is to study trifluridine/tipiracil in combination with other drugs, in particular with biological drugs. We could also study 
the use of trifluridine/tipiracil at different stages of the disease, not only in the advanced/palliative setting but also in earlier phases, such as in first or second-line. Trials in other indications, such as gastric cancer, would also be interesting.

\section{Q Where would you like to see the} management of colorectal cancer in 10 years' time?

I hope that there will be a further improvement in overall survival with a good quality of life due to the use of new drugs and maybe new combinations. I also think that it is important to improve the personalization of treatment for each patient. I hope we will find specific biomarkers that can help us in the choice of tailored treatments and best sequence of treatments for each metastatic colorectal cancer patient.

\section{Q Do you have any closing remarks for our readers?}

We are making much relevant progress, but we need to do more for many of our patients.
For a clinician, the best way to contribute is to collaborate in clinical and translation research.

\section{Financial \& competing interests disclosure}

Professor Falcone has received honoraria for advisory boards and congress participation and funding for research studies from Amgen, Bayer, Roche, Merck, Lilly, Sanofi, and Servier. Dr Salvatore has received honoraria for advisory boards and congress participation from Roche, Bayer, Amgen, Merck, Sanofi, and Servier. The authors have no other relevant affiliations or financial involvement with any organization or entity with a financial interest in or financial conflict with the subject matter or materials discussed in the manuscript apart from those disclosed.

Writing assistance was utilized in the production of this manuscript. We thank Sarah Novack, PhD, who provided editorial assistance on behalf of Servier, France.

\section{Disclaimer}

The opinions expressed in this interview are those of the interviewee and do not necessarily reflect the views of Future Medicine Ltd.

\section{References}

1 Loupakis F, Cremolini C, Masi G et al. Initial therapy with FOLFOXIRI and bevacizumab for metastatic colorectal cancer. N. Engl. J. Med. 371(17), 1609-1618 (2014).

2 Cremolini C, Loupakis F, Antoniotti C et al. FOLFOXIRI plus bevacizumab versus FOLFIRI plus bevacizumab as first-line treatment of patients with metastatic colorectal cancer: updated overall survival and molecular subgroup analyses of the open-label, Phase 3 TRIBE study. Lancet Oncol. 16(13), 1306-1315 (2015).

Mayer RJ, Van Cutsem E, Falcone A et al. Randomized trial of TAS-102 for refractory metastatic colorectal cancer. N. Engl. J. Med. 372(20), 1909-1919 (2015).
4 Lenz HJ, Stintzing S, Loupakis F. TAS-102, a novel antitumor agent: a review of the mechanism of action. Cancer Treat Rev. 41(9), 777-783 (2015)

5 Salvatore L, Rossini D, Moretto R et al. TAS-102 for the treatment of metastatic colorectal cancer. Expert Rev. Anticancer Ther. 15(11), 1283-1292 (2015). 